\title{
Structure-guided design of a high-affinity ligand for a riboswitch
}

\author{
LIN HUANG, JIA WANG, TIMOTHY J. WILSON, and DAVID M.J. LILLEY \\ Cancer Research UK Nucleic Acid Structure Research Group, MSI/WTB Complex, The University of Dundee, Dundee DD1 5EH, United Kingdom
}

\begin{abstract}
We have designed structure-based ligands for the guanidine-II riboswitch that bind with enhanced affinity, exploiting the twin binding sites created by loop-loop interaction. We synthesized diguanidine species, comprising two guanidino groups covalently connected by $C_{n}$ linkers where $n=4$ or 5 . Calorimetric and fluorescent analysis shows that these ligands bind with a 10-fold higher affinity to the riboswitch compared to guanidine. We determined X-ray crystal structures of the riboswitch bound to the new ligands, showing that the guanidino groups are bound to both nucleobases and backbone within the binding pockets, analogously to guanidine binding. The connecting chain passes through side openings in the binding pocket and traverses the minor groove of the RNA. The combination of the riboswitch loop-loop interaction and our novel ligands has potential applications in chemical biology.
\end{abstract}

Keywords: RNA ligand design; molecular recognition; riboregulation; X-ray crystallography

\section{INTRODUCTION}

RNA provides a versatile scaffold for binding small molecule ligands with high selectivity. This is particularly well illustrated by the riboswitches (Roth and Breaker 2009; Serganov and Nudler 2013), cis-acting regulatory elements that occur in the $5^{\prime}$ noncoding regions of (mostly) bacterial mRNA that are widely used to control gene expression. Many classes have now been identified that respond to a range of metabolites including coenzymes, amino acids, purines, and even ions. Atomic resolution structures are available for many riboswitches, and this provides an opportunity to carry out ligand engineering to design new species that will bind with elevated affinity. In this work we have used a structure-guided approach that uses a unique feature of a guanidine riboswitch to create a novel ligand.

The $y k k C$ riboswitches comprise a group of three structurally unrelated riboswitches that bind guanidine. Breaker and colleagues (Nelson et al. 2017) showed that ligand binding to these riboswitches up-regulates the expression of a series of genes whose products either chemically convert guanidine or export it from the cell. Three $y k k C$ types have been identified, called the guanidine-l (Nelson et al. 2017), -II (Sherlock et al. 2017), and -III (Sherlock and Breaker 2017) riboswitches. Crystal structures have been

Corresponding author: d.m.j.lilley@dundee.ac.uk

Article is online at http://www.rnajournal.org/cgi/doi/10.1261/rna. 069567.118. Freely available online through the RNA Open Access option. solved for members of each class (Battaglia et al. 2017; Huang et al. 2017a,b; Reiss and Strobel 2017; Reiss et al. 2017). We have exploited a novel feature of the guanidine-Il riboswitch structure to design a high-affinity ligand.

The guanidine-II riboswitch comprises two stem-loops with $\mathrm{G}+\mathrm{C}$-rich helices and an ACGR ( $=$ A or $\mathrm{G}$ ) tetraloop, connected by a short polynucleotide of $\sim 14$ nt (Sherlock et al. 2017). Individual single stem-loops undergo looploop interaction driven by the cooperative binding of guanidine. Using X-ray crystallography, our laboratory (Huang et al. 2017a) and that of Strobel (Reiss and Strobel 2017) showed that the stem-loops dimerize by loop-loop interaction involving the formation of intermolecular base pairs and triples. Formation of the dimer creates identical binding sites for two guanidine ligands symmetrically. These are bound by donation of guanidine protons to $\mathrm{O} 6$ and N7 of a guanine nucleobase (G9 in our usual numbering scheme [Huang et al. 2017a]), and to nonbridging oxygen atoms of consecutive phosphate groups. At neutral $\mathrm{pH}$ guanidine is protonated, thus having six protons and $D_{3 h}$ symmetry. Four of these protons are involved in specific interactions with the RNA. Protonation confers a positive charge (i.e., it is more properly called the guanidinium cation), and the ligand is stacked upon the guanine nucleobase of the loop-proximal base pair (G6-C11), so that the cation $-\pi$ interaction contributes to the stability of the dimer.

(C) 2019 Huang et al. This article, published in RNA, is available under a Creative Commons License (Attribution 4.0 International), as described at http://creativecommons.org/licenses/by/4.0/. 
In this report, we demonstrate how a structure-guided rational approach can be used to re-engineer the ligand of the guanidine-II riboswitch, exploiting its unusual creation of two binding sites by loop-loop interaction. Sherlock et al. (2017) found that some variants of guanidine with substitution on one nitrogen atom such as methylguanidine and aminoguanidine bound to the guanidine-II riboswitch with affinities that were within a factor of four of guanidine itself. Furthermore, even addition of a butylamine side chain (agmatine) led to retention of binding despite a further loss of affinity. Our crystal structure of the Gloeobacter violaceus guanidine-II riboswitch (Huang et al. 2017a) revealed that the binding pocket had a side opening that might accommodate one or more additional atoms attached to one nitrogen. We diffused these compounds into our crystals, and obtained structures of the riboswitch bound to the modified guanidine species (Huang et al. 2017a). This revealed that the guanidino moiety was bound in exactly the same way as guanidine, and that the additional methyl, amino and butylamine side chains did indeed emerge from the side pocket. In the latter case while the electron density for the guanidine was clear, that for the longer side chain was poorly defined suggesting that it became progressively more mobile as it emerged from the side opening.

The side openings of the two guanidine binding sites in the stem-loop dimer are $\sim 7 \AA$ apart and oriented toward each other on the minor groove side of the loop-loop interface, so we wondered if two guanidino moieties might be covalently linked to create a higher-affinity ligand. Molecular modeling based on our structure of guanidine-bound riboswitch suggested that two guanidine units linked by $\mathrm{C}_{4}$ or $\mathrm{C}_{5}$ polymethylene chains should bind to the riboswitch.

We therefore set out to synthesize $\mathrm{C}_{4-}$ and $\mathrm{C}_{5}$-linked diguanidine species and examine their binding to the $G$. violaceus guanidine-II riboswitch. We have found that both compounds bind to the riboswitch with enhanced affinity and lower stoichiometry. We crystallized the two bound complexes and solved their structures, showing that the linked ligands bind in the anticipated manner.

\section{RESULTS AND DISCUSSION}

\section{Synthesis of diguanidine species}

In order to explore the binding properties of linked guanidines, we synthesized two forms of diguanidine species in which the guanidine moieties are covalently connected by four or five carbon atoms (Fig. 1). For clarity, we term these species diguanidine-
$\mathrm{C}_{4}\left[\mathrm{~N}, \mathrm{~N}^{\prime}\right.$-(butane-1,4-diyl)bis guanidinium, also known as arcaine $]$ and diguanidine- $\mathrm{C}_{5}\left[\mathrm{~N}, \mathrm{~N}^{\prime}\right.$-(pentane-1,5-diyl)bis guanidinium also known as audouine] respectively. The synthetic procedures are detailed in Materials and Methods. In brief, diguanidine- $\mathrm{C}_{4}$ and diguanidine- $\mathrm{C}_{5}$ were synthesized by guanylation of (4-aminobutyl)guanidine (agmatine) and 1,5-diaminopentane (cadaverine) using 1 and 2 molar equivalents of $1-\mathrm{H}$-pyrazole-1-carboxamidine hydrochloride (Bernatowicz et al. 1992), respectively (Supplemental Fig. S1A). The products were characterized by ${ }^{1} \mathrm{H}$ NMR and mass spectrometry (Supplemental Fig. S1B, C), although the crystal structures of the complexes (see below) provide unambiguous evidence that these compounds have the required structure.

\section{Calorimetric analysis of binding of guanidine and diguanidine species to the guanidine-II riboswitch}

We have investigated the binding of guanidine, diguanidine- $C_{4}$, and diguanidine- $C_{5}$ to individual guanidine-II riboswitch stem-loops and a complete riboswitch using isothermal titration calorimetry (ITC) (Fig. 2; Supplemental Fig. S2). First, we have studied the individual P1 and P2 stem-loops derived from $G$. violaceous, for which we have previously determined crystal structures (Huang et al. 2017a). Titration curves are presented in Supplemental Figure S2. The binding of guanidine to the P1 and P2 stem-loops is an exothermic, enthalpy-dominated reaction. Fitting the titration curves gives dissociation constants $\left(K_{d}\right)$ for the binding reaction of $68 \mu \mathrm{M}$ and $66 \mu \mathrm{M}$, respectively, at molar ratios $n=1.9$ and 2.2 (Supplemental Table S1). A stoichiometry of $n=1$ is expected for the binding of guanidine to the isolated stem-loops, but crystal structures reveal a second guanidine binding site near the top of the stem-loop. Reiss and Strobel (2017) observed spermidine and a hydrated magnesium ion bound to the same site, which evidently has a tendency to bind cationic<smiles>N=C(N)N</smiles>

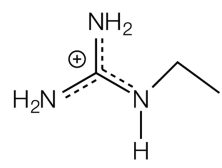

ethylguanidine

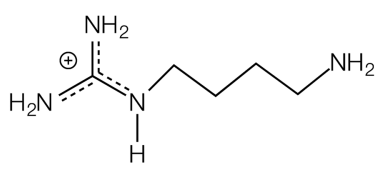

agmatine

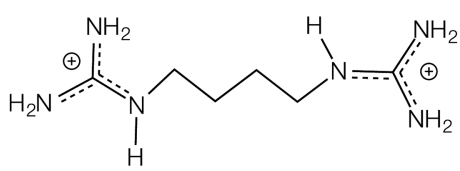

diguanidine- $\mathrm{C}_{4}$

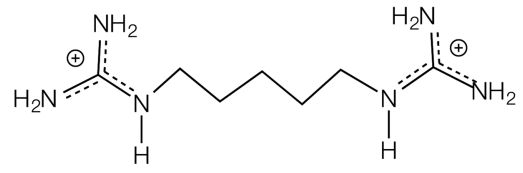

diguanidine- $\mathrm{C}_{5}$

FIGURE 1. Chemical structures of guanidine, modified guanidine species, and diguanidine species discussed in this work. The parental guanidine is shown top left, followed by ethylguanidine and agmatine that are modified by addition of ethyl and butylamine groups, respectively. Below are shown the diguanidine- $C_{4}$ and diguanidine- $C_{5}$ species in which two guanidine groups are connected by $\mathrm{C}_{4}$ and $\mathrm{C}_{5}$ chains, respectively. In all cases, the guanidino moieties are shown as guanidinium cations that are the abundant form at neutral $\mathrm{pH}$. 


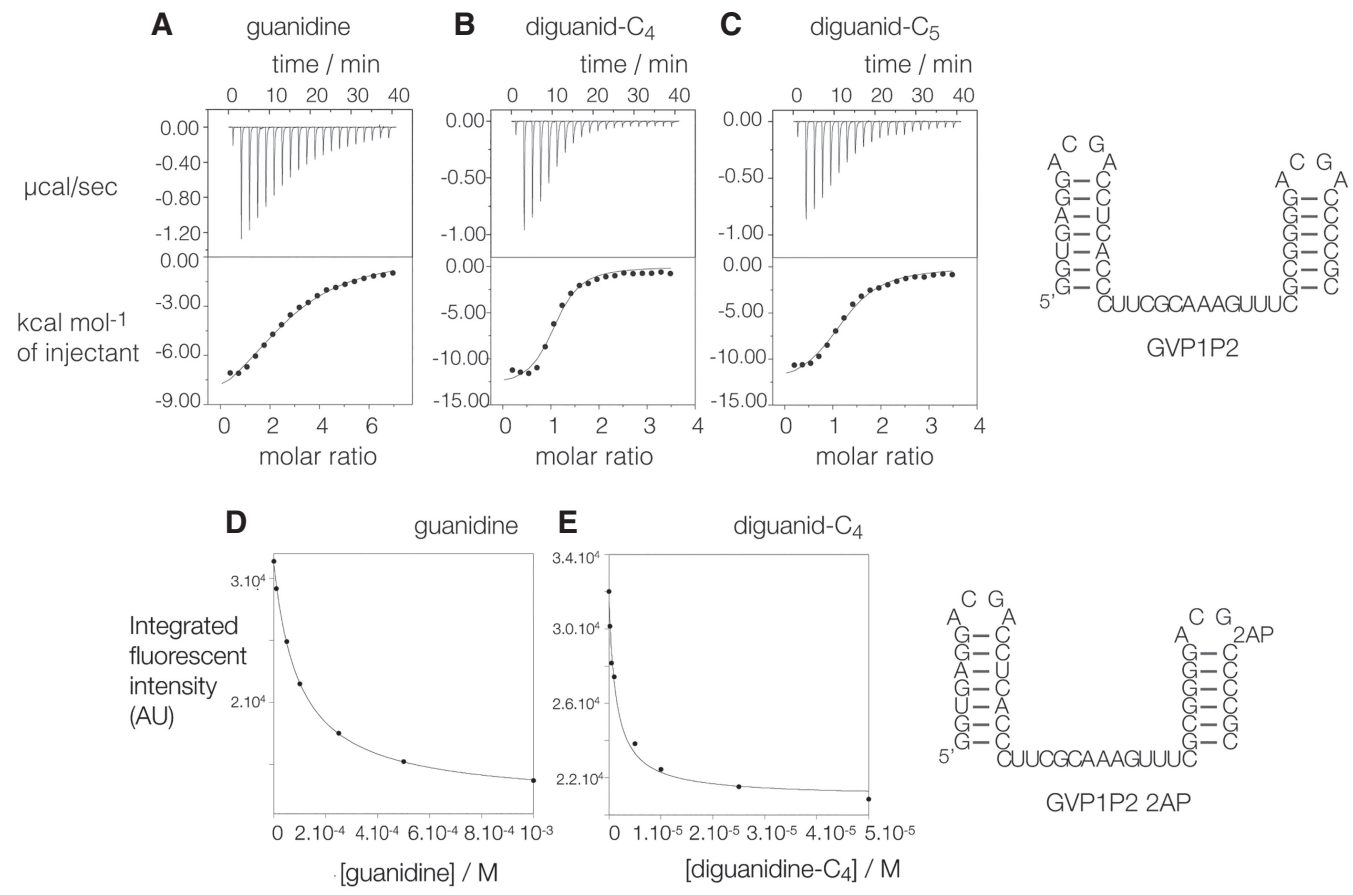

FIGURE 2. Binding of guanidine and diguanidine species to the complete G. violaceous guanidine-ll riboswitch studied by isothermal titration calorimetry and fluorescence spectroscopy. (A-C) Calorimetry. A solution of ligand was titrated into the RNA solution, and the heat evolved was measured as the power required to maintain zero temperature difference with a reference cell. Integration over time gives the heat required to maintain thermal equilibrium between cells. In each case, the upper panel shows the raw data for sequential injections of $2 \mu \mathrm{L}$ volumes (following an initial injection of $0.4 \mu \mathrm{L}$ ) of ligand into $200 \mu \mathrm{L}$ of a $15 \mu \mathrm{M}$ RNA solution in $40 \mathrm{mM} \mathrm{HEPES}$ (pH 7.2), $100 \mathrm{mM} \mathrm{KCl}, 10 \mathrm{mM} \mathrm{MgCl}$. This represents the differential of the total heat (i.e., enthalpy $\Delta H^{\circ}$ under conditions of constant pressure) for each ligand concentration. The lower panels present the integrated heat data fitted to a one-set-of-sites binding model. The thermodynamic parameters calculated are summarized in Supplemental Table S1. Binding was studied using the ligands guanidine $(A)$, diguanidine- $C_{4}(B)$, and diguanidine- $C_{5}(C)$. The sequence of the riboswitch is shown on the right. Titration of individual P1 and P2 stem-loops is shown in Supplemental Figure S2. (D,E) Ligand-induced folding of the guanidine riboswitch studied by 2-aminopurine fluorescence. The GVP1P2 construct used in these experiments (the sequence is shown on the right) contains a single A10 2-aminopurine (2AP) at the $3^{\prime}$ end of the loop. On binding, the ligand loop-loop interaction generates A10-A10' stacking in the crystal, and results in static quenching of 2 -aminopurine fluorescence. Fluorescence emission spectra $\left(\lambda_{\text {excite }}=315 \mathrm{~nm} ; \lambda_{\text {emission }}=340-450 \mathrm{~nm}\right)$ were recorded as a function of added ligand concentration using guanidine and diguanidine- $\mathrm{C}_{4}$. Fluorescence intensity was integrated between 355 and $375 \mathrm{~nm}$, and plotted as a function of guanidine $(D)$ and diguanidine- $C_{4}(E)$ concentration. Ligand binding was also studied by ITC, shown in Supplemental Figure S2.

ligands, including guanidine. The additional binding site may account for the observed molar ratio of $n \sim 2$, in which case it must bind with a similar affinity. Calorimetric titration was repeated for diguanidine- $C_{4}$, giving values of $K_{d}=4.7$ and $5.9 \mu \mathrm{M}$ for $\mathrm{P} 1$ and $\mathrm{P} 2$, respectively, each with a molar ratio of $n=0.4$, close to the expected stoichiometry of 0.5 . Thus, linking the guanidine ligands increases the affinity by an order of magnitude compared to guanidine.

We have further explored the binding of guanidine, diguanidine- $\mathrm{C}_{4}$, and diguanidine- $\mathrm{C}_{5}$ to a complete $\mathrm{G}$. violaceous riboswitch with linked P1 and P2 stem loops using ITC (Fig. 2A-C). As with the individual stem-loops, all three compounds exhibit exothermic binding, with $K_{d}=33,2.2$, and $5.1 \mu \mathrm{M}$, respectively (Supplemental Table S1). Binding exhibited molar ratios of $n=2.7,1.1$ and 1.2 , respectively. The binding affinities for each compound are approximately twofold higher for the complete riboswitch compared to the individual stem-loops. Furthermore, as with the individual stem-loop structures, the diguanidine spe- cies bind an order of magnitude more tightly than guanidine and diguanidine- $\mathrm{C}_{4}$ binds with the highest affinity. The results are consistent with the binding of multiple molecules of guanidine to the riboswitch and a single molecule of the diguanidine species with $\sim 10$-fold higher affinity.

\section{Spectroscopic analysis of binding of diguanidine species to the complete guanidine-II riboswitch}

A feature of the dimerization of the stem-loops observed in the crystal was mutual stacking of the $\mathrm{A} 10$ nucleobases, that is, the 3'-terminal nucleotide of the loop. We have exploited this interaction to construct a spectroscopic probe of the loop-loop interaction between the stem-loops of the complete $G$. violaceous riboswitch. We synthesized a riboswitch in which A10 of the P2 loop was replaced by 2 -aminopurine. This nucleobase is fluorescent, but subject to marked static quenching when stacked with another 
nucleobase. We therefore anticipated that the 2-aminopurine fluorescence intensity might decrease upon binding guanidine or related ligands that induce loop-loop interaction.

The 2-aminopurine-substituted riboswitch was titrated separately with guanidine and diguanidine- $\mathrm{C}_{4}$, recording the emission spectrum of 2-aminopurine excited at $315 \mathrm{~nm}$. Addition of the ligands led to reduced fluorescence. The fluorescence intensity was integrated between 355 and $375 \mathrm{~nm}$, and plotted as a function of ligand concentration (Fig. 2D,E). Addition of guanidine leads to a threefold quenching of 2-aminopurine fluorescence, and fitting the observed intensities to a two-state binding model gives $K_{d}=53 \mu \mathrm{M}$ (Fig. 2D). 2-aminopurine fluorescence was also quenched on addition of diguanidine- $C_{4}$, and fitting the intensity data led to a calculated affinity of $K_{d}=1.4$ $\mu \mathrm{M}$ (Fig. 2E). The data are consistent with an intramolecular loop-loop interaction in the riboswitch, involving stacking of the adenine and 2-aminopurine at the 10 positions leading to quenching of the latter.

We have studied the ligand-induced folding of the 2-aminopurinecontaining riboswitch in response to the addition of guanidine and diguanidine- $\mathrm{C}_{4}$ by ITC (Supplemental Fig. $\mathrm{S} 2 \mathrm{~F}, \mathrm{G})$. The ligands bound with an affinity of $K_{d}=41$ and $6.6 \mu \mathrm{M}$, with molar ratios of $n=4.3$ and 0.79 , respectively.

\section{A crystal structure of the guanidine-II riboswitch bound to ethylguanidine}

Ethylguanidine can be regarded as half of diguanidine- $\mathrm{C}_{4}$, just lacking the central $\mathrm{C}-\mathrm{C}$ bond. The compound was soaked into ligand-free crystals of the $G$. violaceous P1 stem-loop (Supplemental Table S2), and the resulting crystals diffracted to $1.54 \AA$ (Supplemental Table S3). The structure (PDB ID 6HBX) is shown in Figure 3. The structure of the stem-loop is closely similar to that bound to guanidine and methylguanidine (Fig. 3B; Huang et al. 2017a). The guanidine moieties are hydrogen bonded in the normal manner to $\mathrm{O} 6$ and N7 on the Hoogsteen edge of $\mathrm{G} 9$, with two guanidine $\mathrm{N}$ atoms donating hydro-

D
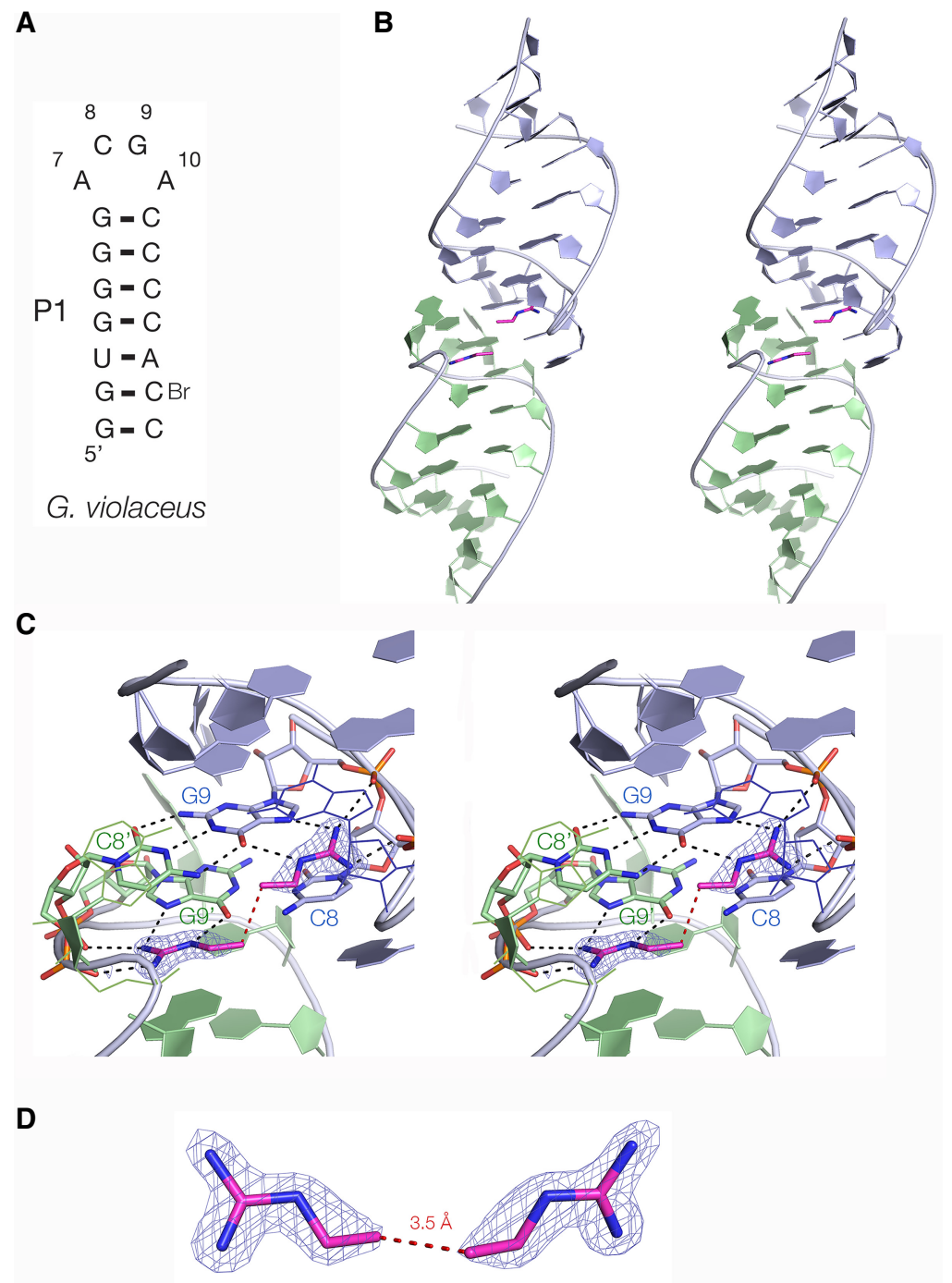

FIGURE 3. Crystal structure of ethylguanidine bound to $G$. violaceous riboswitch P1 stemloop. (A) The sequence of the P1 stem-loop. The nucleotide numbering preserves the A7 to A10 numbering of the loop used previously (Huang et al. 2017b). (B) The overall structure shown in parallel-eye stereoscopic view. The P1 stem-loop forms a dimer by loop-loop interaction; the individual monomeric RNA species are colored here as blue and green. This color scheme is also used in Figure 4. The ethylguanidine molecules are colored magenta. (C) Parallel-eye stereoscopic view of the two bound ethylguanidine molecules bound at the dimer interface, with electron density $\left(2 F_{o}-F_{c}\right)$ contoured at $2 \sigma$ shown for the ligands. The ethylguanidine molecules are hydrogen bonded to G9 and G9' and nonbridging phosphate oxygens of the backbone. $(D)$ The two ethylguanidine molecules with their experimental phasing electron density map contoured at $1 \sigma$. The two terminal carbon atoms are separated by $3.3 \AA$ (broken red line); these would be connected by a single $\mathrm{C}-\mathrm{C}$ bond in diguanidine- $\mathrm{C}_{4}$.

gen bonds to nonbridging $\mathrm{O}$ atoms of successive phosphate groups (Fig. 3C). The ethyl chains emerge from the same side openings as observed with bound methyland aminoguanidine and agmatine (Huang et al. 2017a). The terminal $C$ atoms of the side chain are separated by $3.3 \AA$ in this structure (Fig. 3D). However, the electron density is weak for the terminal atoms and the barrier to rotation about the $\mathrm{N}-\mathrm{C}$ bonds to bring them to within bonding distance $(1.5 \AA)$ should be small. 


\section{A crystal structure of the guanidine-Il riboswitch bound to diguanidine- $\mathrm{C}_{4}$}

Our chemically synthesized diguanidine- $\mathrm{C}_{4}$ was soaked into the $G$. violaceous P1 stem-loop crystals, and the resulting crystals diffracted to $1.66 \AA$ (Supplemental Table S3). The structure was solved (PDB ID 6HBT; Fig. 4) and the position of the diguanidine- $\mathrm{C}_{4}$ ligand is well defined by the electron density map. This demonstrates that the two guanine moieties are bound in the usual manner (Fig. 4B), and are connected by the four-carbon methylene chain just as anticipated. The electron density for the $C_{4}$ linker region is weaker compared to the guanidine groups (Fig. 4C). Moreover the crystallographic B-factors for these
A

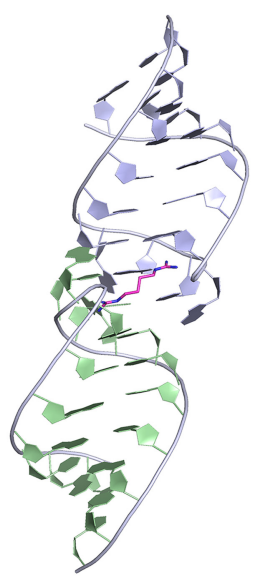

B

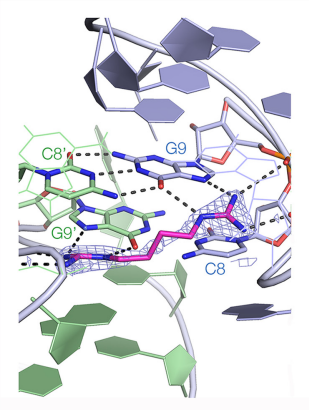

E

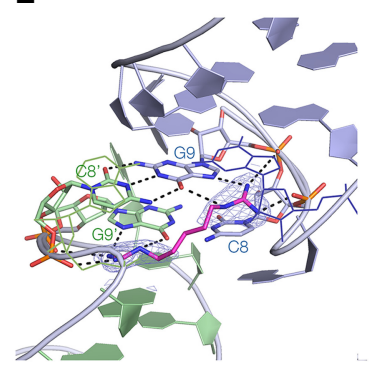

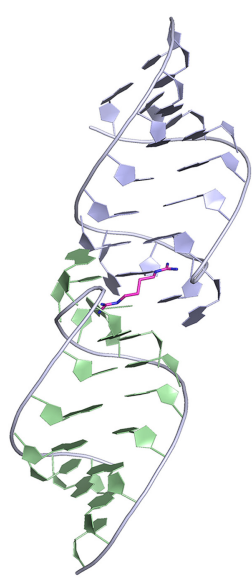

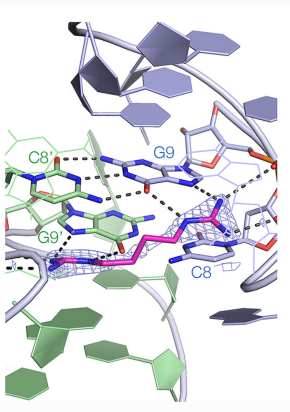

D

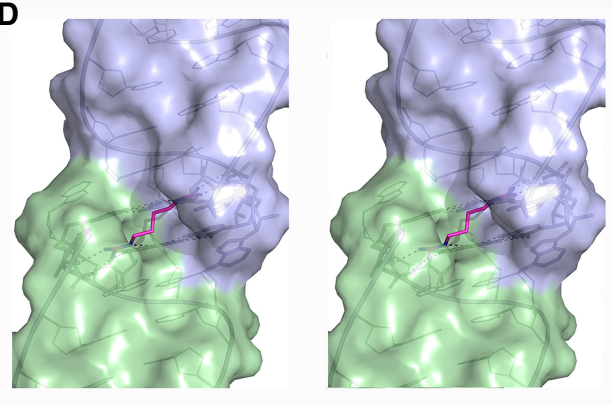

$\mathbf{F}$
C

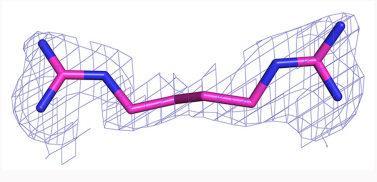

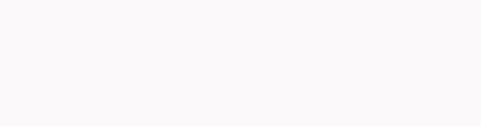
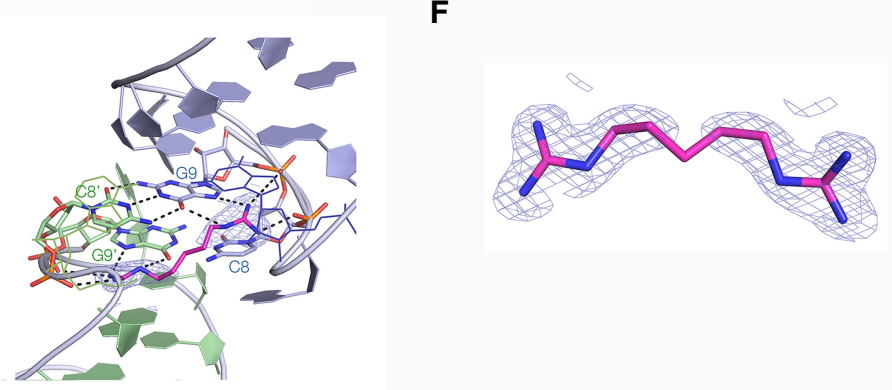

FIGURE 4. Crystal structures of diguanidine- $C_{4}$ and diguanidine- $C_{5}$ bound to $G$. violaceous riboswitch P1 stem-loop. (A) The overall structure of the P1 stem-loop dimer shown in parallel-eye stereoscopic view. The diguanidine- $\mathrm{C}_{4}$ ligand molecule is colored magenta. (B) Parallel-eye stereoscopic view of the diguanidine- $\mathrm{C}_{4}$ molecule bound at the dimer interface. Electron density $\left(2 F_{\mathrm{o}}-F_{\mathrm{c}}\right)$ contoured at $2 \sigma$ is shown for the diguanidine- $\mathrm{C}_{4}$ molecule. Each guanidine moiety is hydrogen bonded to $\mathrm{G} 9$ and $\mathrm{G} 9$ ' and nonbridging phosphate oxygens of the backbone. (C) The diguanidine- $\mathrm{C}_{4}$ with its experimental phasing electron density map contoured at $1 \sigma$. The position of the entire chain is defined, although the density for the central carbon atoms is weaker, indicative of some flexibility in this region. (D) A parallel-eye stereoscopic view of the dimer interface with a surface depicted for the riboswitch with bound diguanidine- $\mathrm{C}_{4}$. The polymethylene chain connecting the guanidine moieties is clearly visible emerging from the side openings of the binding pockets and traversing the minor groove side of the dimer interface. (E) A paralleleye stereoscopic close-up view of the dimer interface with bound diguanidine- $C_{5}$, with the electron density map $\left(2 F_{0}-F_{c}\right)$ for the ligand contoured at $2 \sigma$. Each guanidine moiety is hydrogen bonded to G9 and G9' and nonbridging phosphate oxygens of the backbone. $(F)$ The bound diguanidine- $C_{5}$ molecule shown in isolation, with its experimental phasing electron density map contoured at $1 \sigma$. Electron density for the central carbon atom in the polymethylene linker is not visible, indicative of significant flexibility in the polymethylene chain. 
atoms are significantly higher (average value of 57.5) compared to those of the guanidino- $C$ atoms (46.2). These data collectively suggest a greater flexibility of the $C_{4}$ chain, most likely in a kind of crankshaft rotation. Figure 4D shows that the ligand emerges from the side opening of the binding pocket, traverses the minor groove and enters the binding pocket of the second site.

\section{A crystal structure of the guanidine-II riboswitch bound to diguanidine- $\mathrm{C}_{5}$}

We also soaked our chemically synthesized diguanidine- $\mathrm{C}_{5}$ into the $G$. violaceous P1 stem-loop crystals, and the structure was solved to a resolution of $1.41 \AA$ (PDB ID 6HC5; Fig. 4; Supplemental Table S3). The position of the two guanidine groups is well defined, bound in the normal manner (Fig. 4E). Electron density for the guanidines and the first two $\mathrm{C}$ atoms of the linker is clear, but that for the central $C$ atom cannot be observed at a contour level of $\sigma=1.5$ (Fig. 4F). The B-factor for the central $\mathrm{C}$ atom is 86.0, and the mean value for those flanking it is 50.2. The ligand is clearly bound in the expected manner, but the longer linker is more mobile. Our calorimetric measurements (Supplemental Table S1) show that lower free energy of binding of diguanidine- $C_{5}$ compared to diguanidine- $C_{4}$ is entirely due to a greater $\mathrm{T} \Delta \mathrm{S}$, consistent with a higher conformational entropy of the linker.

\section{Conclusion}

The work described here clearly demonstrates that the two guanidine ligands of the guanidine-II riboswitch can be covalently linked by a chain of four or five methylene carbon atoms that pass through the side openings of the two ligand binding pockets located on the minor groove side of the dimeric riboswitch. The diguanidine ligands are hydrogen bonded into the two binding sites in the same manner as guanidine (Huang et al. 2017a), or ethylguanidine, although with subtle changes of position (Supplemental Fig. S3). The guanidine groups of diguanidine- $\mathrm{C}_{5}$ and ethylguanidine are within $0.2 \AA$, but those of diguanidine- $\mathrm{C}_{4}$ are retracted by $0.4 \AA$. This indicates that the shorter linker may be under some degree of tension.

We have measured the affinity of binding by calorimetry and fluorescence spectroscopy. Although there is some variation in the affinities measured using different methods, the diguanidine ligands bind with affinities that are consistently one order of magnitude higher than that of guanidine. Diguanidine- $\mathrm{C}_{4}$ has a slightly higher affinity than that of diguanidine- $\mathrm{C}_{5}$, most probably because of the greater flexibility of the linking chain in the latter. It is possible that rigidifying the linking chain could further increase binding affinity. The stoichiometry of the binding of the diguanidine ligands is consistent with binding as a single ligand. Thus, the results are entirely consistent with our structural understanding of the guanidine-II riboswitch and provide a new class of higher-affinity ligand.

The work here provides an example of structure-based ligand design using a natural riboswitch. The guanidine-II riboswitch ACGR stem-loop is one of the smallest riboswitches and so easy to combine with other RNA elements. The combination of this with diguanidine ligands has a number of potential applications in chemical and synthetic biology and RNA-based nanotechnology. For these, the diguanidine- $\mathrm{C}_{4}$ has a number of important properties. The compound binds to the riboswitch-derived RNA with higher affinity compared to guanidine, and is a nonnatural compound so chemically orthogonal to cellular metabolism. Importantly, diguanidine- $\mathrm{C}_{4}$ has low toxicity in rats (Ceretta et al. 2008), and is much less toxic than guanidine. This specific ligand-induced RNA interaction could be generally applicable in RNA technology, RNA design, and perhaps RNA-based therapeutics.

\section{MATERIALS AND METHODS}

\section{Synthesis and characterization of diguanidine compounds}

Diguanidine compounds were synthesized by guanylation of amines using 1-H-pyrazole-1-carboxamidine hydrochloride, following procedure B of Bernatowicz et al. (1992) (Supplemental Fig. S1). NMR spectra were recorded using a Bruker Avance DPX 400 spectrometer $\left({ }^{1} \mathrm{H}\right.$ at $\left.400 \mathrm{MHz} ; 9.4 \mathrm{~T}\right)$ using automatic tuning and matching. Chemical shifts $(\delta)$ are expressed in ppm recorded using the residual solvent peak at $4.7 \mathrm{ppm}$ as the internal reference in both cases. Signal splitting patterns are described as triplet $(t)$, quintet, multiplet $(\mathrm{m})$, or a combination thereof. Coupling constants $(J)$ are quoted to the nearest $0.1 \mathrm{~Hz}$. Fast atom bombardment (FAB) mass spectrometry was performed using an Agilent G6470A Triple Quadrupole spectrometer in positive mode of detection. A syringe pump was used to deliver methanol solutions of the diguanidine compounds $(1.0 \mu \mathrm{g} / \mathrm{mL})$ with a flow rate of 0.5 $\mathrm{mL} / \mathrm{min}$. A stainless-steel capillary was held at a potential of $3.0 \mathrm{kV}$. Nitrogen was used as nebulizer gas at a flow rate of $7.0 \mathrm{~L} / \mathrm{min}$, pressure $40 \mathrm{PSI}$ at $350^{\circ} \mathrm{C}$. Reported spectra are the averages of 15 scans using 500 msec accumulation time.

$N, N^{\prime}$-(butane-1,4-diyl)bis guanidinium (diguanidine- $C_{4}$ ). Agmatine sulfate ( $85 \mathrm{mg}, 370 \mu \mathrm{mol}), 1-\mathrm{H}$-pyrazole-1-carboxamidine hydrochloride $(55 \mathrm{mg}, 370 \mu \mathrm{mol})$, and $0.75 \mathrm{~mL}$ of $1.0 \mathrm{M} \mathrm{Na}_{2} \mathrm{CO}_{3}$ were stirred overnight at room temperature. The white precipitate was collected and washed three times with $1 \mathrm{~mL} \mathrm{MeOH} / \mathrm{H}_{2} \mathrm{O}(1: 1)$ then dried in vacuo to yield $54 \mathrm{mg}(53 \%)$. ${ }^{1} \mathrm{H}$ NMR : $3.13(4 \mathrm{H}, \mathrm{m}$, $\left.\left(\mathrm{CH}_{2}\right)_{2}\right), 1.56\left(4 \mathrm{H}, \mathrm{m},\left(\mathrm{CH}_{2}\right)_{2}\right)$ (Supplemental Fig. S2A). FAB mass spectrometry gave a molecular mass of 173.10 (calculated 173.25) (Supplemental Fig. S1B).

$N, N^{\prime}$-(pentane-1,5-diyl)bis guanidinium (diguanidine- $C_{5}$ ). Cadaverine hydrochloride (100 mg, $590 \mu \mathrm{mol}), 1-\mathrm{H}$-pyrazole-1carboxamidine hydrochloride $(170 \mathrm{mg}, 1.18 \mathrm{mmol}$ ) and $2.4 \mathrm{~mL}$ of $1.0 \mathrm{M} \mathrm{Na}_{2} \mathrm{CO}_{3}$ were stirred overnight at room temperature. The white precipitate was collected and washed three times with $1 \mathrm{~mL} \mathrm{MeOH} / \mathrm{H}_{2} \mathrm{O}(1: \mathrm{l})$ then dried in vacuo to yield $110 \mathrm{mg}$ 
(74\%). ${ }^{1} \mathrm{H}$ NMR : $3.10\left[4 \mathrm{H}, \mathrm{t}, \mathrm{J} 6.3 \mathrm{~Hz},\left(\mathrm{CH}_{2}\right)_{2}\right], 1.53$ [4H, quintet, J $\left.6.9 \mathrm{~Hz},\left(\mathrm{CH}_{2}\right)_{2}\right], 1.36-1.28\left(2 \mathrm{H}, \mathrm{m}, \mathrm{CH}_{2}\right)$ (Supplemental Fig. S2B). FAB mass spectrometry gave a molecular mass of 187.10 (calculated 187.25) (Supplemental Fig. S1C).

\section{Synthesis of RNA oligonucleotides}

RNA oligonucleotides were synthesized using solid-phase $t$ BDMS phosphoramidite chemistry (Beaucage and Caruthers 1981) as described in Wilson et al. (2001), implemented on an Applied Biosystems 394DNA/RNA synthesizer. Oligonucleotides containing 5-bromocytidine (ChemGenes) were deprotected in a $25 \%$ ethanol/ammonia solution for $36 \mathrm{~h}$ at $20^{\circ} \mathrm{C}$. The oligonucleotide containing 2-aminopurine (Glen Research) was deprotected in 1:1 ammonia/methylamine solution for $20 \mathrm{~min}$ at room temperature followed by $10 \mathrm{~min}$ at $65^{\circ} \mathrm{C}$. All oligoribonucleotides were redissolved in $100 \mu \mathrm{L}$ of anhydrous DMSO and $125 \mu \mathrm{L}$ triethylamine trihydrofluoride (Aldrich) to remove t-BDMS groups, and agitated at $65^{\circ} \mathrm{C}$ in the dark for $2.5 \mathrm{~h}$. After cooling on ice for $10 \mathrm{~min}$, the RNA was precipitated with $1 \mathrm{~mL}$ of butanol, washed twice with $70 \%$ ethanol and suspended in double-distilled water.

RNA was purified by gel electrophoresis in polyacrylamide under denaturing conditions in the presence of $7 \mathrm{M}$ urea. The fulllength RNA product was visualized by UV shadowing. The band was excised and electroeluted using an Elutrap Electroelution System (GE Healthcare) into $45 \mathrm{mM}$ Tris-borate (pH 8.5), $5 \mathrm{mM}$ EDTA buffer for $8 \mathrm{~h}$ at $200 \mathrm{~V}$ at $4^{\circ} \mathrm{C}$. The RNA was precipitated with ethanol, washed once with $70 \%$ ethanol, and suspended in double-distilled water.

\section{Isothermal titration calorimetry}

ITC titrations were performed at $298 \mathrm{~K}$ using an ITC-200 microcalorimeter (GE Healthcare). RNA solutions $(30-60 \mu \mathrm{M})$ were prepared by diluting concentrated stocks into the binding buffer containing $40 \mathrm{mM}$ HEPES ( $\mathrm{pH}$ 7.2), $100 \mathrm{mM} \mathrm{KCl}, 10 \mathrm{mM} \mathrm{MgCl}_{2}$. Guanidine and diguanidine compounds were prepared in the same binding buffer with a concentration of 0.5-1 mM. Solutions were degassed for 2-5 min before loading. The sample cell was filled with $200 \mu \mathrm{L}$ of RNA. Guanidine or diguanidine was injected in a volume of $0.4 \mu \mathrm{L}$ for the first injection and $2 \mu \mathrm{L}$ for the next 19 injections using a computer-controlled $40 \mu \mathrm{L}$ microsyringe with an injection interval of $120 \mathrm{sec}$. Titration of ligands into the binding buffer or titration of the binding buffer into the RNA solution resulted in negligible evolution of heat. Integrated heat data were analyzed using a one-set-of-sites model in MicroCal Origin following the manufacturer's instructions. The first data point was excluded in analysis. The binding parameters $\Delta H$ (reaction enthalpy change in cal $\mathrm{mol}^{-1}$ ), $K$ (binding constant in $\mathrm{M}^{-1}$ ), and $n$ (bound ligands per RNA) were variables in the fit. The binding free energy $\Delta G$ and reaction entropy $\Delta S$ were calculated using the relationships $\Delta G=$ $-R T \ln K$, where $R=1.987 \mathrm{cal} \mathrm{mol}^{-1} \mathrm{~K}^{-1}, T=298 \mathrm{~K}$ and $\Delta G=\Delta H$ $-T \Delta S$. The dissociation constant $K_{d}$ was calculated as $1 / K$.

\section{Fluorescence spectroscopy}

Fluorescence spectra were recorded in $10 \mathrm{mM}$ Tris- $\mathrm{HCl}(\mathrm{pH}$ 8.0), $50 \mathrm{mM} \mathrm{NaCl}$, and $10 \mathrm{mM} \mathrm{MgCl}_{2}$ at $25^{\circ} \mathrm{C}$ using an SLM-Aminco
8100 fluorimeter. The spectra were corrected for lamp fluctuations and instrumental variations, and polarization artifacts were avoided by crossing excitation and emission polarizers at $54.7^{\circ}$. Steady-state fluorescence emission spectra were recorded between $330 \mathrm{~nm}$ and $460 \mathrm{~nm}$ in $1 \mathrm{~nm}$ intervals with excitation at $315 \mathrm{~nm}$. Spectra were integrated between 355 and $375 \mathrm{~nm}$.

\section{X-ray crystallography}

The $G$. violaceus P1 stem-loop RNA sequence used for crystallization was $\left(5^{\prime}-3^{\prime}\right)$ GGUGGGGACGACCCCA $(\mathrm{BrC}) \mathrm{C}$ where $\mathrm{BrC}$ is 5-bromocytosine. A solution of $1 \mathrm{mM}$ RNA in $5 \mathrm{mM}$ HEPES $(\mathrm{pH}$ 7.6), $100 \mathrm{mM} \mathrm{KCl}$ was heated to $95^{\circ} \mathrm{C}$ for $1 \mathrm{~min}$. The solution was slowly cooled to $20^{\circ} \mathrm{C}$ and $\mathrm{MgCl}_{2}$ added to a final concentration of $2 \mathrm{mM}$. Ligands were soaked into crystals of the ligand-free P1 RNA using the conditions indicated in Supplemental Table S2. All the crystals were cryoprotected using mother liquid with an additional 25\%-30\% glycerol.

Diffraction data were collected on beamlines 104 and 103 of Diamond Light Source (Harwell, UK). Data were processed by XIA2 (Winter et al. 2018). The resolution cutoff for the data was determined by examining by $\mathrm{CC} 1 / 2$ and density map as described previously (Karplus and Diederichs 2012). Initial phase information were acquired from the SAD data by locating the bromine atoms with Autosol in the PHENIX suite. Models were adjusted manually using Coot (Emsley et al. 2010) and subjected to several rounds of adjustment and optimization using Coot, phenix.refine, and PDB_REDO (Joosten et al. 2014). Model geometry and the fit to the electron density maps were monitored with MOLPROBITY (Chen et al. 2010) and the validation tools in Coot. The unbiased electron density maps were generated through Br-SAD phasing and density modification by Phenix AutoSol. Details of data collection and refinement statistics for the crystallographic data are shown in Supplemental Table S3.

\section{SUPPLEMENTAL MATERIAL}

Supplemental material is available for this article.

\section{ACKNOWLEDGMENTS}

We thank Saira Ashraf, Lauren Webster, and De Lin for oligonucleotide synthesis, NMR spectroscopy, and mass spectrometry, respectively, and Cancer Research UK for financial support (program grant A18604). We thank Diamond for synchrotron time and the Wellcome Trust for the in-house diffractometer.

Received November 11, 2018; accepted December 28, 2018.

\section{REFERENCES}

Battaglia RA, Price IR, Ke A. 2017. Structural basis for guanidine sensing by the $y k k C$ family of riboswitches. RNA 23: 578-585. doi: 10.1261/rna.060186.116

Beaucage SL, Caruthers MH. 1981. Deoxynucleoside phosphoramidites-a new class of key intermediates for deoxypolynucleotide synthesis. Tetrahedron Lett 22: 1859-1862. doi:10.1016/S00404039(01)90461-7 
Bernatowicz MS, Wu Y, Matsueda GR. 1992. 1H-pyrazole-1-carboxamidine hydrochloride: an attractive reagent for guanylation of amines and its application to peptide synthesis. J Org Chem 57: 2497-2502. doi:10.1021/jo00034a059

Ceretta AP, Camera K, Mello CF, Rubin MA. 2008. Arcaine and MK801 make recall state-dependent in rats. Psychopharmacology 201: 405-411. doi:10.1007/s00213-008-1304-7

Chen VB, Arendall WB III, Headd JJ, Keedy DA, Immormino RM, Kapral GJ, Murray LW, Richardson JS, Richardson DC. 2010 MolProbity: all-atom structure validation for macromolecular crystallography. Acta Crystallogr D Biol Crystallogr D66: 12-21. doi:10.1107/S0907444909042073

Emsley P, Lohkamp B, Scott WG, Cowtan K. 2010. Features and development of Coot. Acta Crystallogr D Biol Crystallogr 66: 486-501. doi:10.1107/S0907444910007493

Huang L, Wang J, Lilley DMJ. 2017a. The structure of the guanidineII riboswitch. Cell Chem Biol 24: 695-702.e2. doi:10.1016/ j.chembiol.2017.05.014

Huang L, Wang J, Wilson TJ, Lilley DMJ. 2017b. Structure of the guanidine III riboswitch. Cell Chem Biol 24: 1407-1415e2. doi: 10.1016/j.chembiol.2017.08.021

Joosten RP, Long F, Murshudov GN, Perrakis A. 2014. The PDB REDO server for macromolecular structure model optimization. IUCrJ 1: 213-220. doi:10.1107/S2052252514009324

Karplus PA, Diederichs K. 2012. Linking crystallographic model and data quality. Science 336: 1030-1033. doi:10.1126/science.1218231

Nelson JW, Atilho RM, Sherlock ME, Stockbridge RB, Breaker RR. 2017. Metabolism of free guanidine in bacteria is regulated by a widespread riboswitch class. Mol Cell 65: 220-230. doi:10.1016/ j.molcel.2016.11.019

Reiss CW, Strobel SA. 2017. Structural basis for ligand binding to the guanidine-II riboswitch. RNA 23: 1338-1343. doi:10.1261/ rna.061804.117

Reiss CW, Xiong Y, Strobel SA. 2017. Structural basis for ligand binding to the guanidine-I riboswitch. Structure 25: 195-202. doi: 10.1016/j.str.2016.11.020

Roth A, Breaker RR. 2009. The structural and functional diversity of metabolite-binding riboswitches. Annu Rev Biochem 78: 305334. doi:10.1146/annurev.biochem.78.070507.135656

Serganov A, Nudler E. 2013. A decade of riboswitches. Cell 152: 1724. doi:10.1016/j.cell.2012.12.024

Sherlock ME, Breaker RR. 2017. Biochemical validation of a third guanidine riboswitch class in bacteria. Biochemistry 56: 359-363. doi:10.1021/acs.biochem.6b01271

Sherlock ME, Malkowski SN, Breaker RR. 2017. Biochemical validation of a second guanidine riboswitch class in bacteria. Biochemistry 56: 352-358. doi:10.1021/acs.biochem.6b01270

Wilson TJ, Zhao ZY, Maxwell K, Kontogiannis L, Lilley DMJ. 2001. Importance of specific nucleotides in the folding of the natural form of the hairpin ribozyme. Biochemistry 40: 2291-2302. doi: 10.1021/bi002644p

Winter G, Waterman DG, Parkhurst JM, Brewster AS, Gildea RJ, Gerstel M, Fuentes-Montero L, Vollmar M, Michels-Clark T, Young ID, et al. 2018. DIALS: implementation and evaluation of a new integration package. Acta CrystallogrD Struct Biol74:85-97. doi:10.1107/ S2059798317017235 

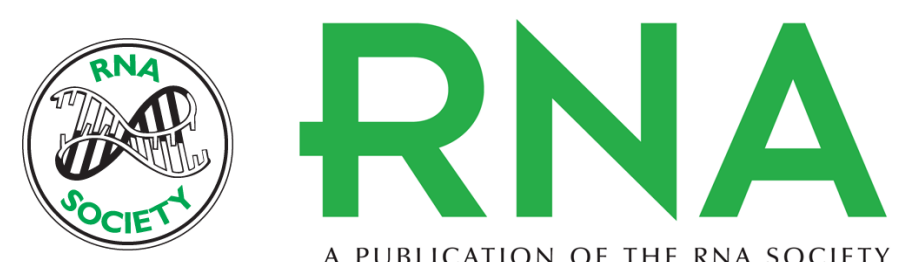

A PUBLICATION OF THE RNA SOCIETY

\section{Structure-guided design of a high-affinity ligand for a riboswitch}

Lin Huang, Jia Wang, Timothy J. Wilson, et al.

RNA 2019 25: 423-430 originally published online January 4, 2019

Access the most recent version at doi:10.1261/rna.069567.118

Supplemental Material

References

Open Access Freely available online through the RNA Open Access option.

Creative This article, published in $R N A$, is available under a Creative Commons License Commons License

Email Alerting Service

http://rnajournal.cshlp.org/content/suppl/2019/01/04/rna.069567.118.DC1

This article cites 19 articles, 3 of which can be accessed free at: http://rnajournal.cshlp.org/content/25/4/423.full.html\#ref-list-1 (Attribution 4.0 International), as described at http://creativecommons.org/licenses/by/4.0/.

Receive free email alerts when new articles cite this article - sign up in the box at the top right corner of the article or click here. 